\title{
REVIEW
}

\section{High-speed Imaging at 3 Tesla: A Technical and Clinical Review with an Emphasis on Whole-brain 3D Imaging}

\author{
Shinji Naganawa ${ }^{1 *}$, Hisashi Kawai ${ }^{1}$, Hiroshi Fukatsu ${ }^{1}$, Takeo Ishigaki ${ }^{1}$, \\ Tomomi Komada ${ }^{1}$, Katsuya Maruyama ${ }^{2}$, and Osamu TAKIZAWA ${ }^{2}$ \\ ${ }^{1}$ Department of Radiology, Nagoya University School of Medicine \\ 65 Tsurumai-cho, Shouwa-ku, Nagoya 466-8550, Japan \\ ${ }^{2}$ Siemens-Asahi Medical Technologies Ltd.
}

(Received January 12, 2005; Accepted February 2, 2005)

\begin{abstract}
Improvements to the inherently high specific-absorption rate (SAR) of high-speed imaging at $3 \mathrm{~T}$ are necessary in order to render this method clinically feasible. Various efforts have been undertaken to improve the associated hardware and software. In this review, we focus on whole-brain isotropic 3D imaging with a turbo spin-echo sequence with variable flip-angle echo trains (3D-TSE-VFL) and present its technical and clinical features. This sequence can be used to acquire images of various contrasts including $\mathrm{T}_{2}$-weighted, fat-suppressed $\mathrm{T}_{2}$-weighted, fluid-attenuated inversion recovery (FLAIR), fat-suppressed FLAIR, and STIR (short tau inversion recovery). Various aspects of 3D-TSE-VFL are discussed, including CSF (cerebrospinal fluid) and metal artifacts, STIR contrast, small-part visualization other than brain, and the possibility of serial subtraction. Some images from clinical cases are presented.
\end{abstract}

\section{Keywords: brain, isotropic, high resolution, ultra-high field, variable flip angle}

\section{Introduction}

\section{Current state of imaging at 1.5 Tesla}

Various applications such as BOLD (blood oxygen level-dependent) functional MR (magnetic resonance) imaging of the brain, diffusion- and perfusion-weighted imaging, and spectroscopy demand a high signal-to-noise ratio (SNR). In many cases, the SNR at $1.5 \mathrm{~T}$ is not sufficient for these applications.

With recent developments in parallel imaging techniques, this SNR shortfall has become even more significant. In addition, sensitivity to the minute susceptibility differences required for BOLD contrast, as well as the chemical shift separation required for uniform fat suppression or spectroscopy, are also lacking at $1.5 \mathrm{~T}$. Therefore, the desire for main fields of $3 \mathrm{~T}$ or above is increasing, not only among researchers but also among clinical radiologists. ${ }^{1,2}$

High-speed imaging at 3 Tesla

The most significant issue related to high-speed MR imaging at $3 \mathrm{~T}$ is the SAR (specific absorption

\footnotetext{
*Corresponding author, Phone: +81-52-744-2327, Fax: + 81-
} 52-744-2335, E-mail: naganawa@med.nagoya-u.ac.jp rate), especially in body applications and particularly in comparison to that of $1.5 \mathrm{~T} .{ }^{3}$ Even with head applications, SAR limitations are frequently encountered during turbo spin-echo sequences with long echo trains, true-FISP-type (fast imaging steady-state precession) sequences with high flip angles, and conventional multi-slice spin-echo sequences with $T_{1}$ weighting. SAR is four times higher at $3 \mathrm{~T}$ than at $1.5 \mathrm{~T}$. Accommodating current SAR regulations sometimes requires an increase in repetition time, a reduction in the flip angle, or a reduction in the number of slices. These compromises may alter image contrast, significantly reduce the clinical utility of $3 \mathrm{~T}$ scanners, or both. To address these SAR issues, machine vendors are taking steps to produce more efficient RF (radio frequency) transmit coils designed for specific regions such as the abdomen and heart. Parallel imaging techniques such as SENSE $^{4,5}$ (sensitivity encoding for fast MRI) and SMASH (simultaneous acquisition of spatial harmonics $)^{6-8}$ can also help to reduce SAR by reducing the echo-train length of turbo spin-echo sequences. However, SAR reduction through efficient coils and parallel imaging is still insufficient in many cases. The most important technical development required for the clinical 
feasibility of $3 \mathrm{~T}$ imaging is novel turbo spin-echo sequences using variable flip angles in their refocusing RF pulses while maintaining image contrast and spatial resolution comparable to those of conventional turbo spin-echo with constant 180-degree refocusing pulses. Techniques such as the hyperecho sequence, ${ }^{9}$ the prescribed signal evolution 3D sequence with variable flip-angle echo train, ${ }^{10-12}$ transitions between pseudo steady states (TRAPS), ${ }^{13}$ and some variations of these are capable of significantly reducing SAR to a greater extent than is conventional turbo spin-echo. The focus of this review is whole-brain isotropic 3D imaging with the prescribed signal evolution sequence developed by Mugler, including technical and clinical discussions of our experiences.

Technical aspects of whole-brain isotropic 3D imaging using a prescribed-signal-evolution turbospin-echo sequence with a variable-flip-angle echo train (3D-TSE-VFL)

A hyper-echo sequence employs an RF pulse with a 180-degree flip angle at the center of the echo train, while refocusing pulses with lower flip angles are used elsewhere in the echo train. ${ }^{9}$ Refocusingpulse flip angles are designed to be symmetric about the center 180-degree pulse; thus, the strongest hyper-echo appears as the $(2 n+1)^{\text {th }}$ echo if the 180 degree pulse was used as the $(n+1)^{\text {th }}$ pulse. Hyperecho sequences can typically reduce SAR by a factor of 2 to 3 while maintaining a given signal-tonoise ratio.

The prescribed signal-evolution sequence developed by Mugler ${ }^{12}$ is a 3D turbo spin-echo sequence with variable-flip-angle refocusing pulses (3D-TSEVFL). As with the hyper echo, it uses refocusing pulses with lower flip angles in the echo train; however, the sequence design concept differs from that of a hyper-echo sequence. 3D-TSE-VFL is designed for whole-brain isotropic imaging; thus, the echo train tends to be quite long (about 220) in order to cover the whole brain in a clinically feasible scan time, with an effective echo time of around $350 \mathrm{~ms}$. Unlike a hyper-echo sequence, 3D-TSE-VFL does not typically employ a 180degree pulse, and the echo train design is not symmetric. The echo-train flip angles in 3D-TSEVFL begin with relatively high angles of about 160 degrees, gradually decrease to about 45 degrees, increase to about 80 degrees, and again gradually decrease to about 20 degrees. This complicated sequence design has two purposes: to improve $T_{2}$ contrast between white matter and gray matter during the first high-flip-angle period; and to maintain a constant echo magnitude of brain parenchyma during the next gradually increasing period to reduce blurring of brain tissue. The SAR is usually about one-tenth that of conventional 3D-TSE with constant 180-degree flip angles. An important difference is that constant 180-degree flip-angle 3D-TSE produces hydrographic contrast with an echo-train length of 220 and effective TE of $350 \mathrm{~ms}$, while $3 \mathrm{D}-\mathrm{TSE}-\mathrm{VFL}$ provides excellent $\mathrm{T}_{2}$ contrast between gray and white matter. This is also partly due to the contribution of stimulatedecho pathways. ${ }^{13}$

This sequence is compatible with a pre-inversion pulse and a fat-suppression pulse allowing $T_{2}-$ weighted, fat-suppressed $\mathrm{T}_{2}$-weighted, FLAIR, fatsuppressed FLAIR, and STIR contrasts. Figure 1 shows an example of a typical image.

\section{Materials and Methods}

\section{Sequence parameters}

All images shown in this article were obtained with a 3T MR scanner ("Trio"; Siemens AG, Erlangen, Germany) with a maximum gradient amplitude of $40 \mathrm{mT} / \mathrm{m}$ and a maximum slew rate of $200 \mathrm{mT} / \mathrm{m} / \mathrm{ms}$ equipped with a receive-only 8channel phased-array head coil. A body coil was used for RF transmission.

The parameters for the $\mathrm{T}_{2}$-weighted $3 \mathrm{D}$ turbo spin-echo scan with variable-flip-angle echo train were as follows: a repetition time of $3,500 \mathrm{~ms}$, effective echo time of $353 \mathrm{~ms}$, echo train length of 221 , and echo spacing of $3.13 \mathrm{~ms}$ for 160 sagittal $1-\mathrm{mm}$ thick slices covering the whole brain with a $256-\mathrm{mm} \times 221-\mathrm{mm}$ field of view $(1 \mathrm{~mm} \times 1 \mathrm{~mm} \times$ $1 \mathrm{~mm}$ isotropic voxels). The matrix was $221 \times 256 \times$ 160 with a read-out bandwidth of $930 \mathrm{~Hz} /$ pixel. The number of excitations was one, yielding a scan time of $9 \min 25 \mathrm{~s}$. STIR-contrast 3D imaging can be obtained by adding a pre-inversion pulse with an inversion time of $180 \mathrm{~ms}$. Fat-suppressed $\mathrm{T}_{2}$-weighted 3D imaging can be obtained by adding a frequency-selective fat-suppression pulse to a $\mathrm{T}_{2}$-weighted $3 \mathrm{D}$ sequence.

The parameters for 3D-FLAIR were as follows: a repetition time of $6,000 \mathrm{~ms}$, effective echo time of $353 \mathrm{~ms}$, and inversion time of 2,200 ms. The matrix was $221 \times 256 \times 160$ with a read-out bandwidth of $930 \mathrm{~Hz} /$ pixel and 6/8 partial Fourier on the sliceselect axis. The number of excitations was one, yielding a scan time of $12 \mathrm{~min} 8 \mathrm{~s}$. For evaluating 3D-FLAIR, axial- and sagittal-reformatted images were generated with a slice thickness and inter-slice gap identical to 2D-FLAIR images obtained for comparison.

The parameters for 2D-FLAIR were as follows: a repetition time of $9,000 \mathrm{~ms}$, effective echo time of 

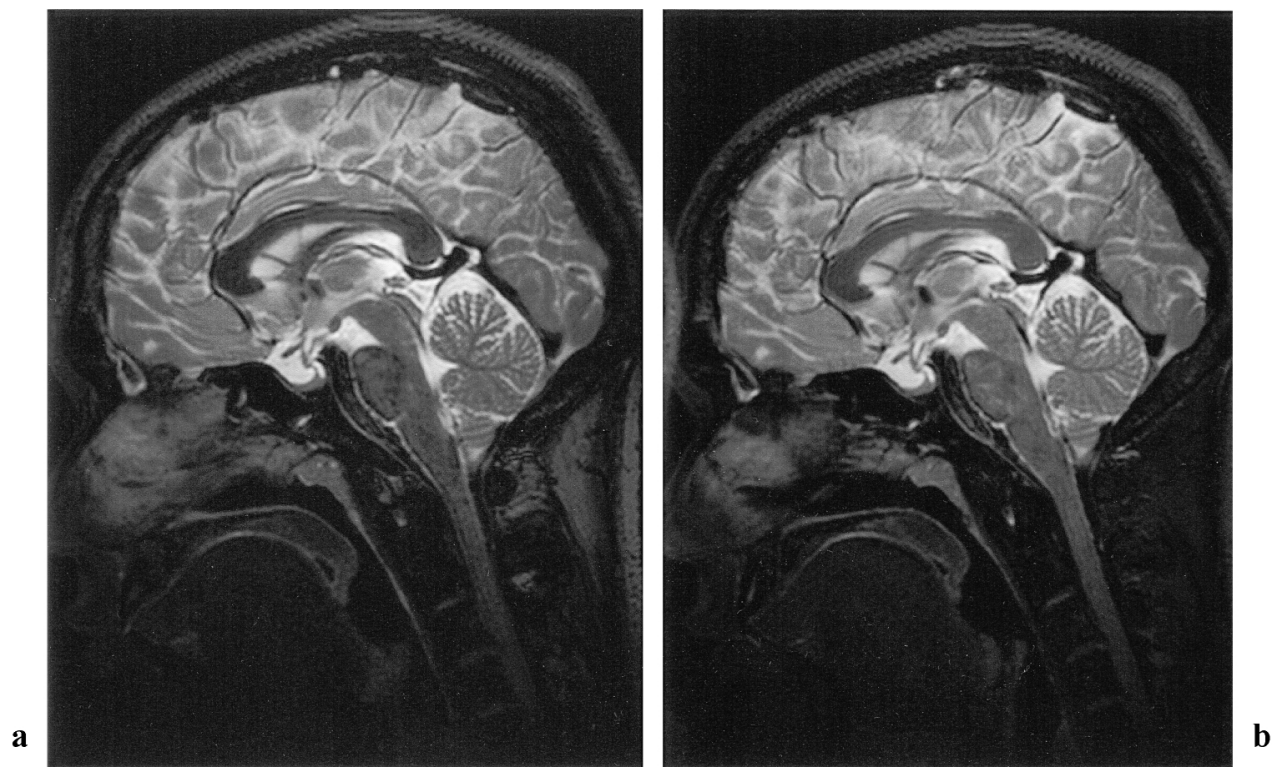

Fig. 1. 3D-TSE-VFL images of the whole brain of a healthy 27-year-old man (a) STIR sagittal image (TR 3500, TE 354, TI 160)

(b) Fat-suppressed $T_{2}$-weighted image (TR 3500, TE 354)

Both images were acquired with 1-mm cubic voxels. Note that gray-white matter contrast is better in (a).

$106 \mathrm{~ms}$ with flow compensation in the slice direction, inversion time of $2,500 \mathrm{~ms}$, echo train length of 15 , echo spacing of $11.8 \mathrm{~ms}$, flip angle of 120 degrees for refocusing pulses to reduce SAR, and 26 slices with 4-mm thickness, 0.4-mm gap, and $230-\mathrm{mm}$ square field of view. The matrix size was $480 \times 640$ with a read-out bandwidth of $227 \mathrm{~Hz}$ / pixel. The images were obtained in two orientations: axially, parallel to the AC-PC line, and sagittally covering the whole brain. The number of excitations was one, yielding a scan time of $5 \mathrm{~min}$ $26 \mathrm{~s}$ (approximately $11 \mathrm{~min}$ to obtain both axial and sagittal images). An acceleration factor of 2 was applied using the parallel imaging technique GRAPPA (generalized autocalibrating partially parallel acquisitions), ${ }^{8}$ an extension of SMASH. ${ }^{6,7}$ The thickness of the inversion slab was $6 \mathrm{~mm}$ to reduce the CSF in-flow artifact; therefore, the 26 slices were divided into two interleaving slice groups to avoid cross-talk between successive inversion pulses. A hyper-echo option was employed. In this review, we evaluate 3D-TSE-VFL with regard to CSF and metal artifacts, STIR contrast, small-part visualization other than the brain, and the possibility of serial subtraction. Some images from clinical cases are presented.

\section{Results and Discussion}

\section{CSF artifacts: 2D- versus 3D-FLAIR}

The FLAIR sequence is part of a routine protocol for brain MRI. ${ }^{14}$ Many studies have noted subtle findings in FLAIR images. ${ }^{15-20}$ Subtle highsignal areas in CSF have been reported to be a sign of subarachnoid hemorrhage ( $\mathrm{SAH})$, meningitis, leptomeningeal dissemination, acute infarction, and moyamoya disease. ${ }^{16}$ Arterial hyperintensity in the middle cerebral artery has been reported to be a sign of acute occlusion or flow reduction in cases of acute stroke. ${ }^{17}$ Thus, high-signal artifacts caused by CSF in-flow or high signal in vessels can result in misdiagnosis during reading of 2D-FLAIR images. $^{21-23}$ It has been reported that most flow artifacts tend to be worse at $3 \mathrm{~T}$ than at $1.5 \mathrm{~T} .{ }^{24}$ Thus, it is quite important to reduce CSF artifacts in FLAIR at 3T.

Many efforts have been made to reduce in-flow artifacts $^{25}$ in 2D-FLAIR images. ${ }^{21-23,26,27}$ These methods can be divided into two groups-selective and non-selective-according to the type of inversion pulses used.

The most common method used with selective inversion pulses is to widen the inversion region, on the order of $50 \%$ wider than the slice thickness, to diminish inflow of non-inverted magnetization. ${ }^{25}$ With this approach, it is necessary to divide and interleave the acquisition to avoid cross-talk of 

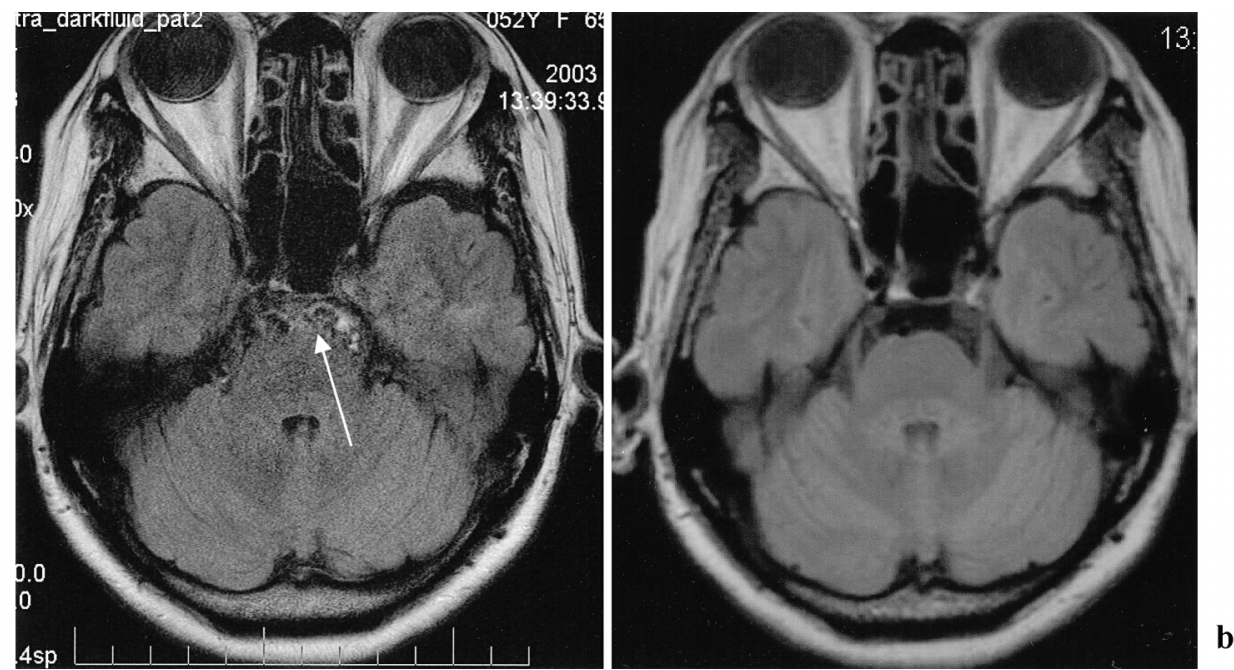

Fig. 2. Comparison of CSF flow artifacts between 2D-FLAIR (a) and 3DFLAIR contrast (b). Note that the inflow artifact is prominent in the prepontine cistern (a, arrow). No artifact is seen with 3D-FLAIR contrast (b).

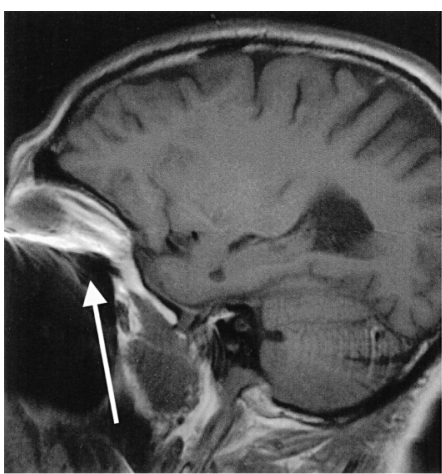

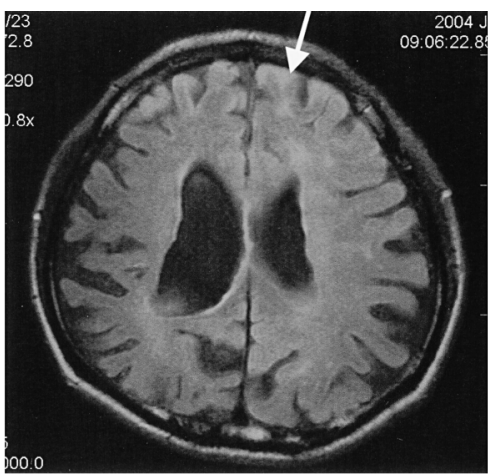

b

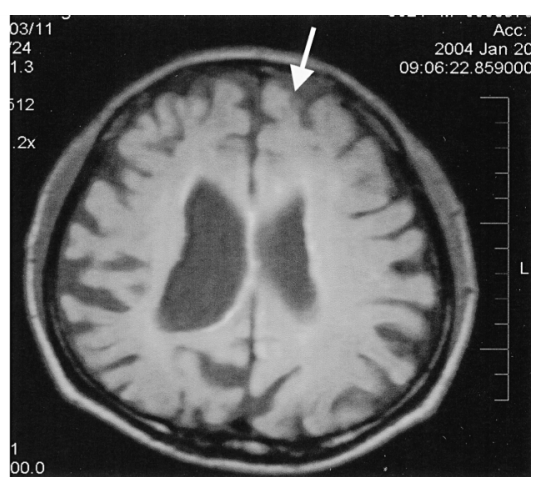

Fig. 3. A 62-year-old man in a post-systemic chemotherapy state for malignant lymphoma. This patient complains of headache.

A severe dental-metal artifact appears in sagittal $\mathrm{T}_{1}$-weighted images (arrow, a). A high-signal area is apparent in the frontal subarachnoid space with 2D-FLAIR contrast (b, arrow), but not with 3D-FLAIR contrast (c, arrow). The high signal with 2D-FLAIR contrast is a metallic artifact mimicking a subarachnoid hemorrhage.

inversion pulses; therefore, the acquisition time must be doubled. The use of wider inversion regions, however, does not necessarily reduce the artifact. Bakshi et al. ${ }^{25}$ using an optimized 2D-FLAIR pulse sequence, reported CSF-related artifacts with high signal intensity in the fourth ventricle in $50 \%$ of subjects. Ascending slice ordering combined with a saturation pulse positioned inferior to the slices might be a simple approach to reducing CSF artifacts.

Among the non-selective inversion methods for eliminating CSF flow artifacts, the most prevalent employs a non-slice-selective inversion pulse together with a technique called "K-space reordered by inversion-time for each slice position"
(KRISP) to achieve constant contrast in multi-slice acquisitions. ${ }^{22}$ This can provide good suppression of the CSF signal; however, the scan time may be as long as $13 \mathrm{~min}$ for 20 axial slices. ${ }^{22}$ Another method employing non-selective inversion pulses in 2D-FLAIR was reported by Tanaka et al. ${ }^{28}$ The imaging volume was divided into sections with a different inversion time for each section. All sections were acquired twice, once in ascending order and once in descending order, and were averaged to achieve approximately the same contrast properties for each section. Because a non-selective inversion pulse was used, the degree of artifact suppression should be comparable to that of our single-slab 3D technique. However, the method 
$\mathbf{a}$

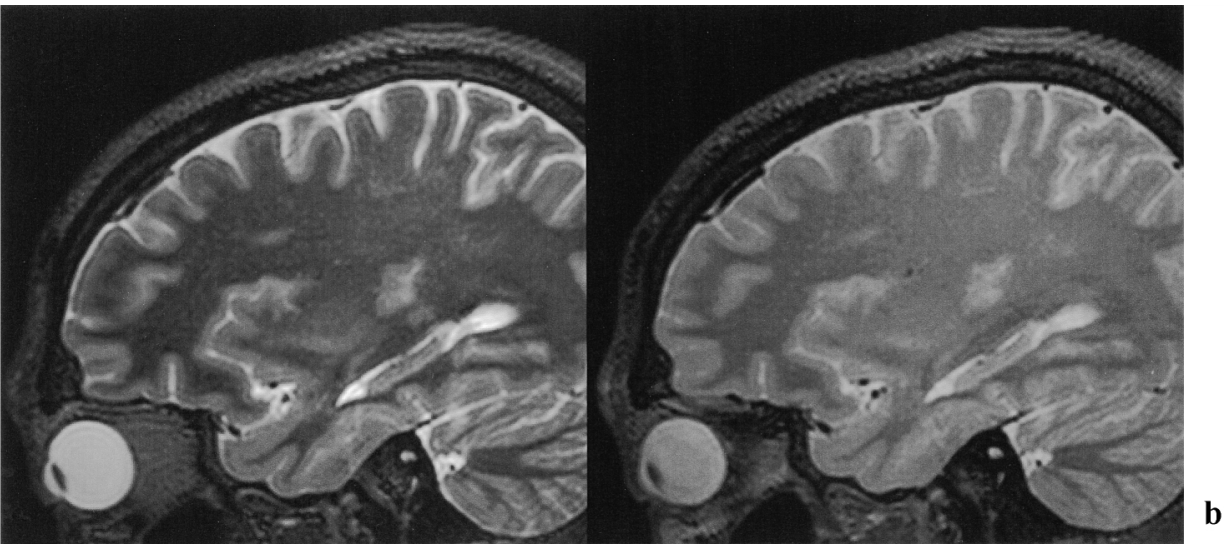

Fig. 4. A healthy 27-year-old man. Comparison of gray-/white-matter contrast between 3D-STIR-contrast (a) and fat-suppressed $\mathrm{T}_{2}$-weighted 3D imaging (b).

The gray-/white-matter contrast-to-noise ratio is 1.6 times higher with 3DSTIR-contrast than with fat-suppressed $\mathrm{T}_{2}$-weighted 3D images.

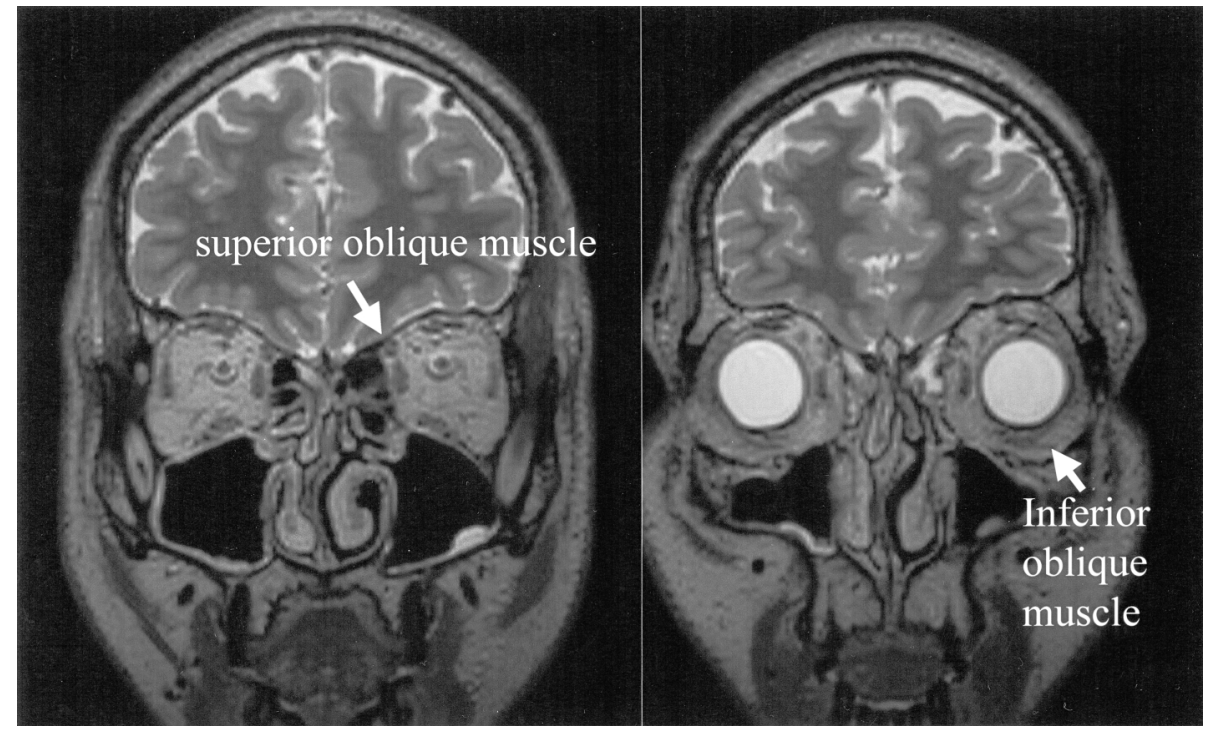

Fig. 5. Coronal-reformatted $\mathrm{T}_{2}$-weighted $3 \mathrm{D}$ imaging of a healthy 32 -year-old man. We can appreciate structures other than the brain such as external muscle, the optic nerves, and mucosa in the maxillary sinus.

used by Tanaka et al. is inferior in time efficiency and susceptible to misregistration between the two acquisitions due to patient motion. Other methods use modified RF pulses. One method reported to be useful is the application of tailored radio-frequency (TRF) pulses in an echo-amplitude stabilization scheme with flip-angle adjustment and increased interleaving acquisition factor in a fast spin-echo sequence. ${ }^{29}$ Use of adiabatic inversion pulses can also reduce CSF artifacts. ${ }^{21}$

Although 3D-FLAIR with non-selective inversion is desirable, a clinically realistic scan time cannot be achieved with conventional 3D turbo spin-echo with an echo-train length of 15-25.
Mugler et al. introduced a variable flip-angle 3D turbo spin-echo sequence for brain imaging with non-selective RF pulses. ${ }^{12}$ They have already shown the positive effect of 3D-FLAIR for the suppression of CSF in-flow artifacts in axial images at $1.5 \mathrm{~T} .^{26}$ We have shown that the CSF-related artifact is less prominent with 3D-FLAIR than with 2D-FLAIR at 3T, not only for axial images but also for sagittal images (Fig. 2) ${ }^{30}$ However, CSF is not completely dark with 3D-FLAIR, as it is with 2D-FLAIR, probably due to the shorter TR and TI in 3DFLAIR.

\section{Metal artifact}

With 2D-FLAIR, dental-metal artifacts cause 
a
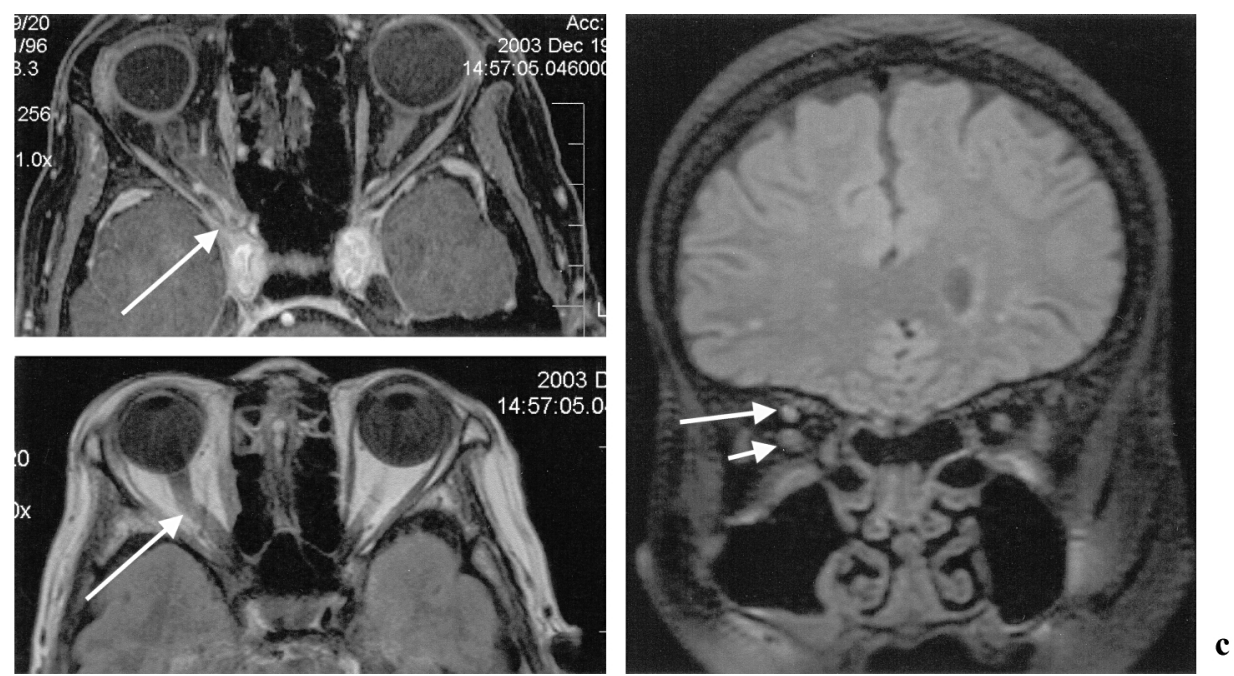

b

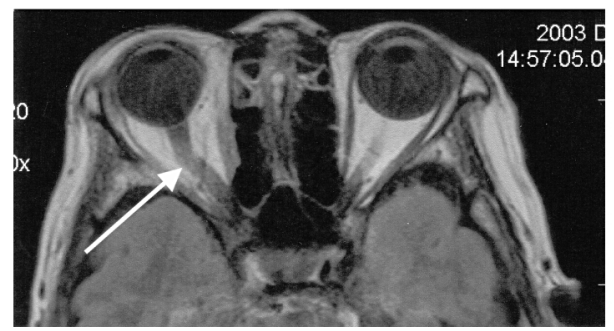

c

Fig. 6. A 67-year-old woman with orbital pain. A small enhanced lesion at the right orbital apex is seen in post-contrast $3 \mathrm{D} \mathrm{T}_{1}$-weighted images with water excitation (a, arrow). The right optic nerve is swelling and edematous in a 2D-FLAIR contrast image (b, arrow). A coronal-reformatted fat-suppressed 3D-FLAIR contrast image shows congestion of the right superior ophthalmic vein (arrow, c) and swelling of the right optic nerve (short arrow, c).

high-signal CSF in the frontal area, mimicking subarachnoid hemorrhage (SAH). Dental metal can partially disrupt inversion of CSF by the sliceselective pre-inversion pulse. 3D-FLAIR, however, employs a non-selective pre-inversion pulse; thus, 3D-FLAIR is less susceptible to dental-metal artifacts (Fig. 3).

\section{STIR contrast}

STIR contrast is useful for the detection of pathologies in or near fat tissue. For brain imaging, STIR contrast provides excellent delineation of gray-white contrast. We measured the contrast-tonoise ratio between gray matter and white matter in healthy volunteers. The mean contrast-to-noise ratio was 1.6 times higher with STIR contrast images than with fat-suppressed $\mathrm{T}_{2}$-weighted images (Fig. 4).

Small-part visualization of features other than the brain

\section{Orbita}

With coronal MPR (multi- planar reconstruction), external orbital muscles can be appreciated (Fig. 5). Fat-suppressed FLAIR imaging enables the assessment of the optic nerve and intra-orbital structures such as the superior ophthalmic vein (Fig. 6).

\section{Inner ear}

Four nerve branches in the internal auditory canal are frequently visualized (Fig. 7). Thus, small vestibular schwannoma may be detected without contrast administration. However, the spatial reso-

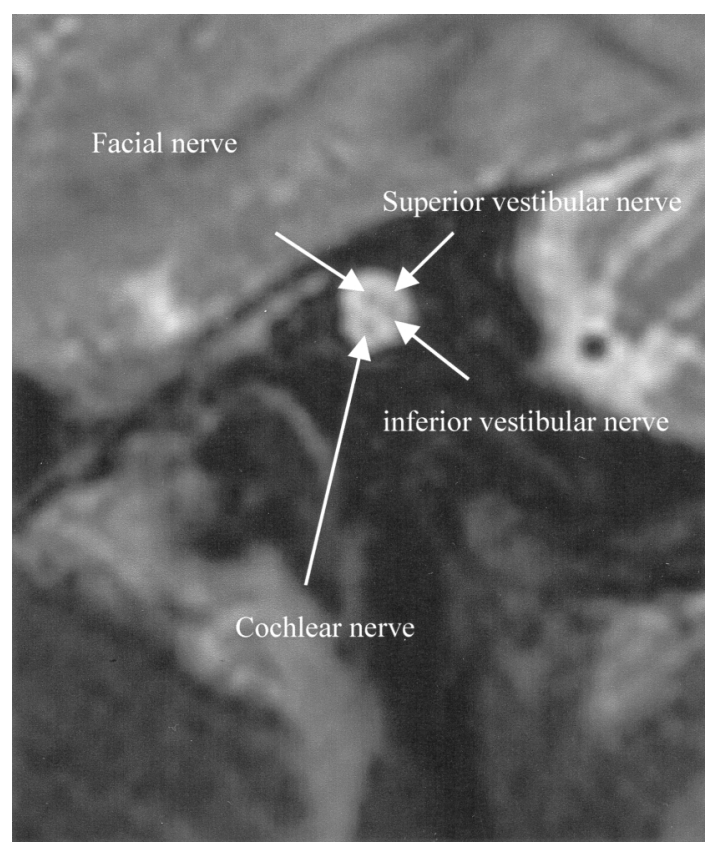

Fig. 7. A healthy 32-year-old man. Sagittal $\mathrm{T}_{2}$-weighted $3 \mathrm{D}$ imaging shows four nerves in the internal auditory canal.

lution may not be sufficient for evaluation of intralabyrinthine pathology.

Flow void of arteries

In $\mathrm{T}_{2}$-weighted $3 \mathrm{D}$ images, a CSF flow void in the prepontine cistern is prominent, hindering delineation of the vertebrobasilar artery. However, the shape of the basilar top can be appreciated in 

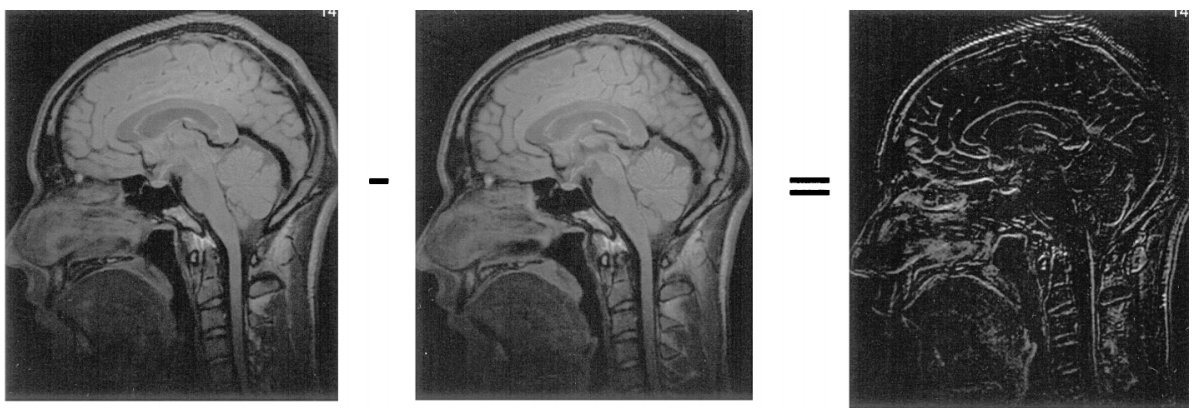

Subtraction image of 3D-FLAIR

a
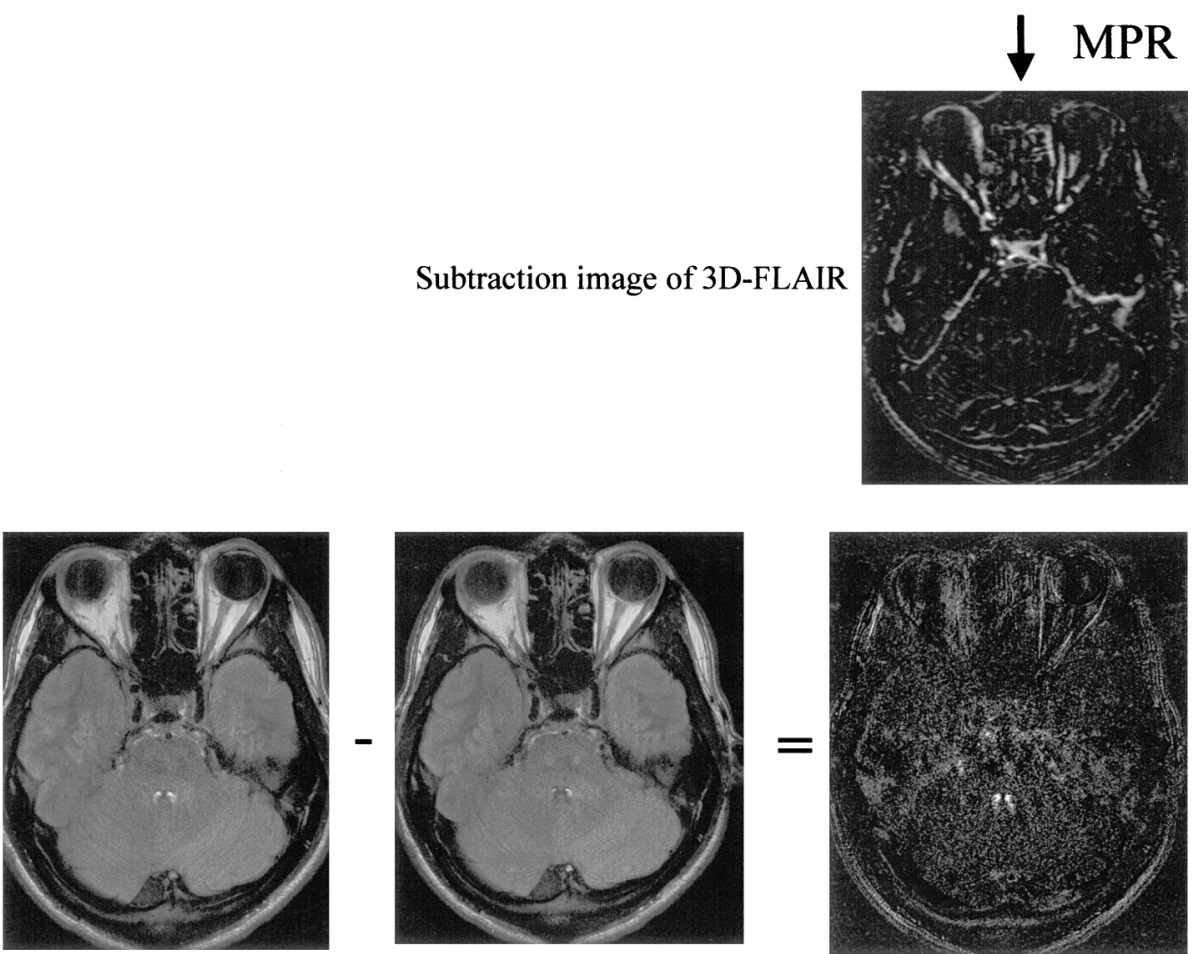

b

Subtraction image of 2D-FLAIR
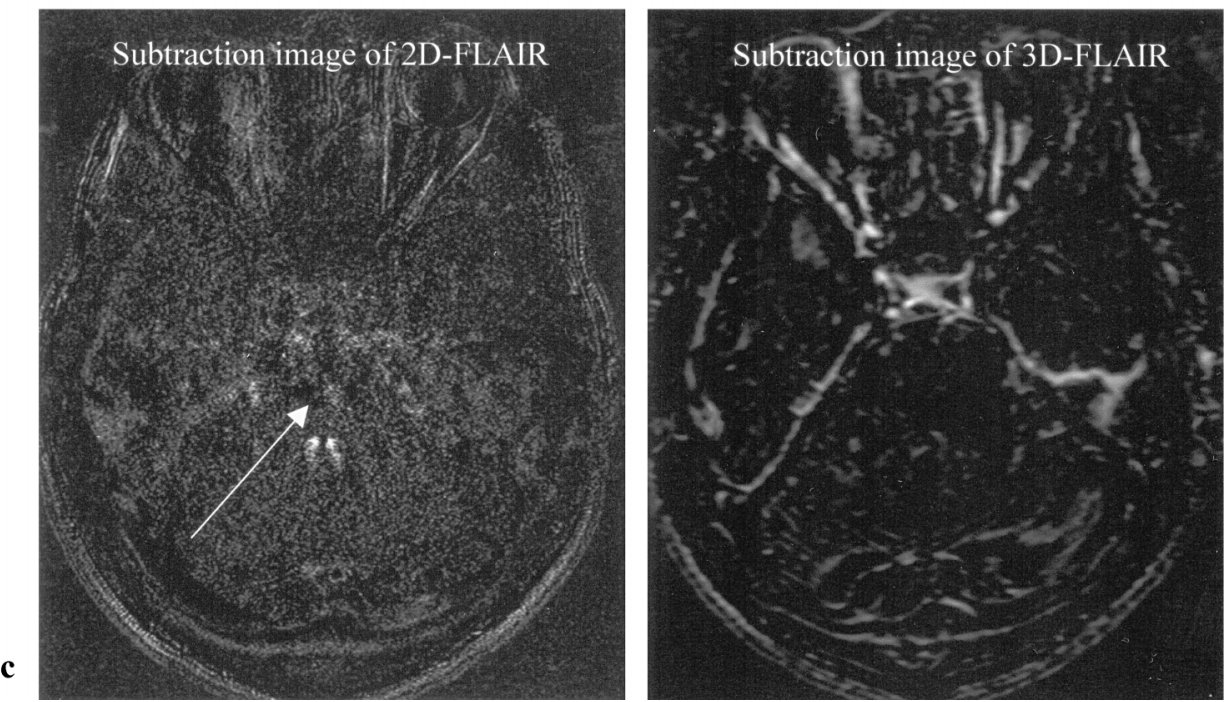

Fig. 8. An initial trial of serial subtraction of two FLAIR data sets obtained at different times in a healthy volunteer.

(a) Schematic diagram for the subtraction of 3D-FLAIR data.

(b) Schematic diagram for the subtraction of 2D-FLAIR data.

(c) Comparison of the subtraction images resulting from 2D- (left) and 3D-FLAIR (right). Note that a residual CSF flow artifact is prominent in the brain stem in the 2D-FLAIR subtraction image (arrow). No artifact is seen in the 3D-FLAIR subtraction image. 


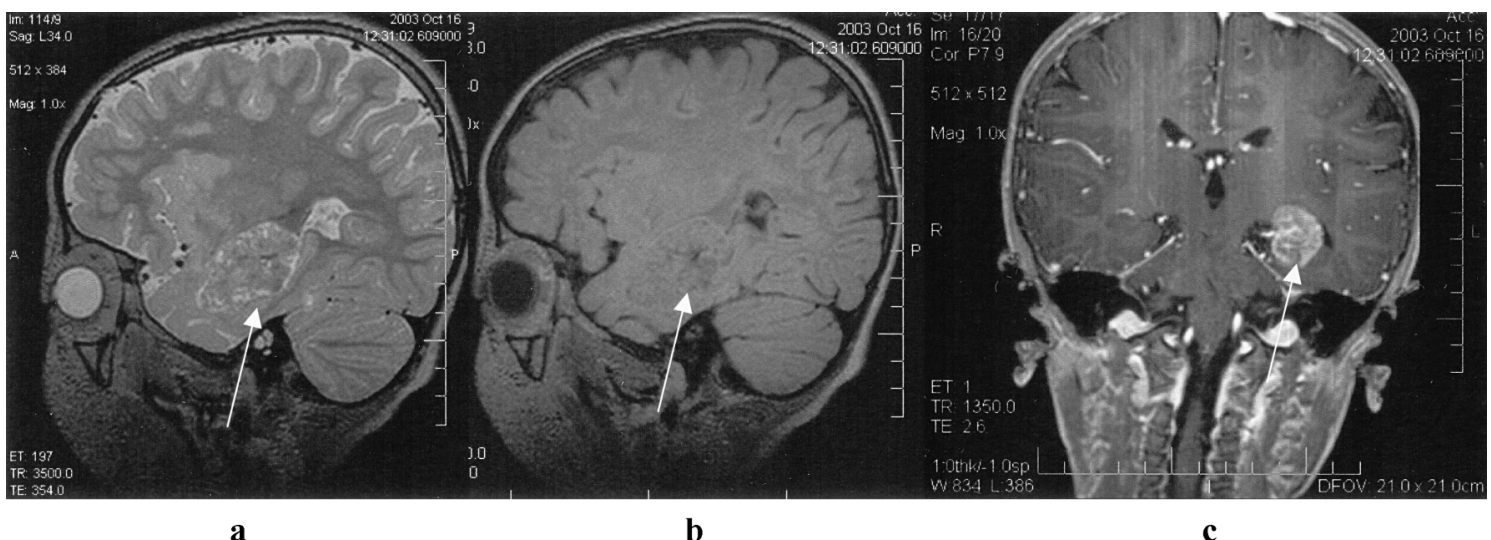

a

b

c

Fig. 9. A 4-year-old girl with choroid plexus papilloma in the left lateral ventricle. $T_{2}$-weighted 3D image (a), 3D-FLAIR contrast image (b), and post-contrast-enhanced $\mathrm{T}_{1}$-weighted 3D-MP-RAGE image (c). The papillary tumor can be appreciated in all images (arrows). Whole-brain isotropic 3D imaging can be obtained with various types of contrast.

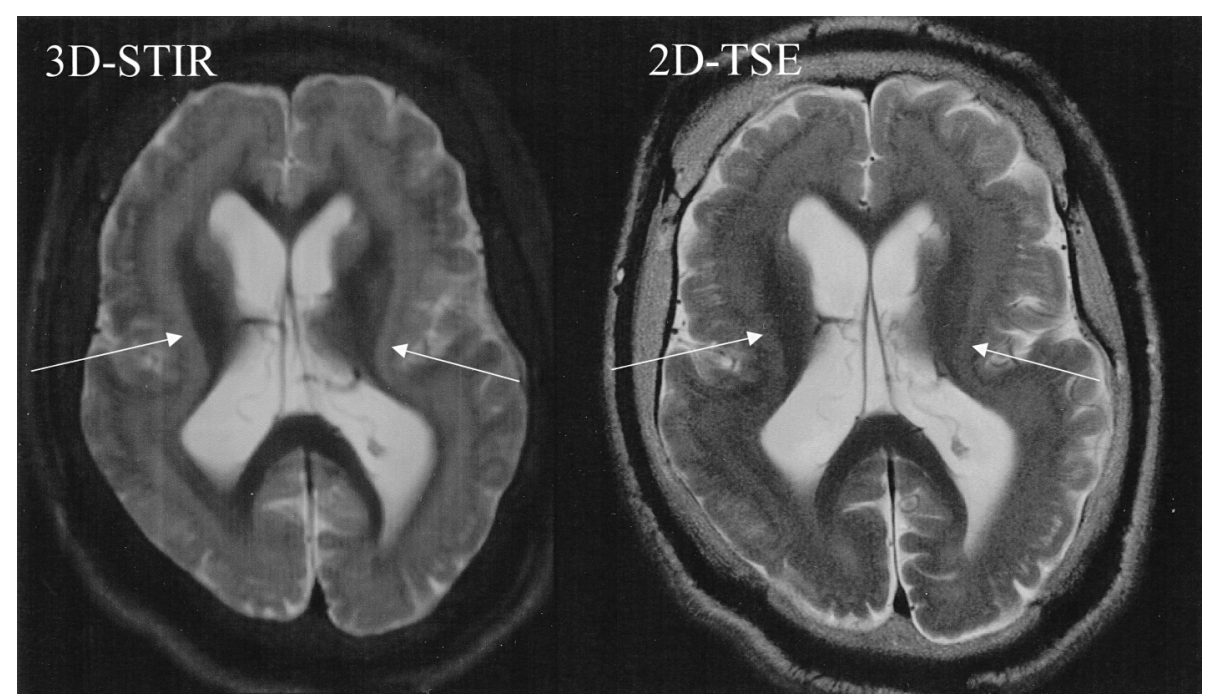

Fig. 10. A 25-year-old man with band heterotopia. Although in-plane resolution is better in $2 \mathrm{D}-\mathrm{T}_{2}$-weighted images, gray-white contrast is far better in $3 \mathrm{D}$ STIR contrast images. Thus, the delineation of band-like ectopic gray matter is much more prominent with axial-reformatted 3D-STIR contrast than with 2DTSE sequence (arrows).

many cases. This adds useful information regarding the basilar top when 3D time-of-flight MR angiography provides only equivocal findings.

\section{Feasibility of serial subtraction}

Gapless slices and the lack of CSF-related artifacts with 3D-FLAIR make the subtraction of serially acquired studies possible with simple registration, even when the studies are acquired in separate imaging sessions. ${ }^{31}$ Our initial trial of serial subtraction on a healthy volunteer is shown in Fig. 8. In the subtraction of 2D-FLAIR images obtained during two separate sessions, the CSFartifact residual overlapping the brainstem is far more prominent than in the subtraction of $3 \mathrm{D}$ FLAIR images.

Clinical cases

Some clinical examples are presented to show the clinical utility of 3D-TSE-VFL sequences (Figs. 9-12).

\section{Possibility of further speed increases}

\section{Parallel imaging}

Currently, the scan time for 3D-TSE-VFL is relatively long, in the range of $8-13 \mathrm{~min}$. The resulting isotropic data allow multiplanar recon- 


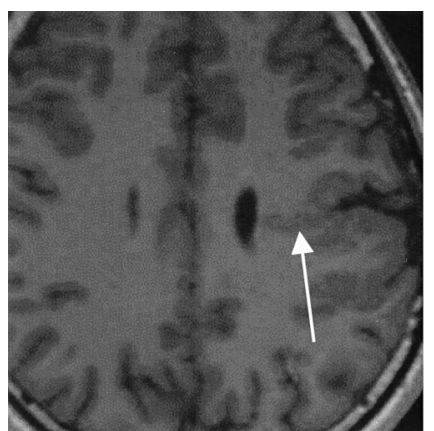

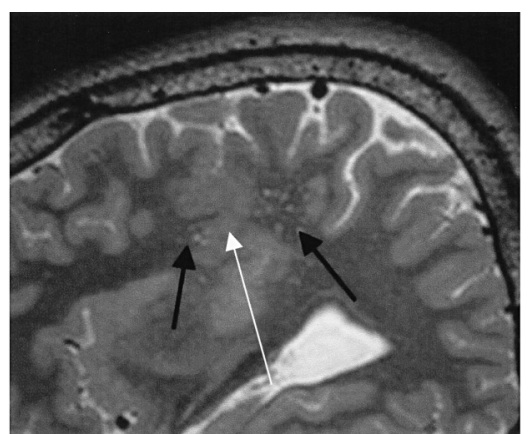

b

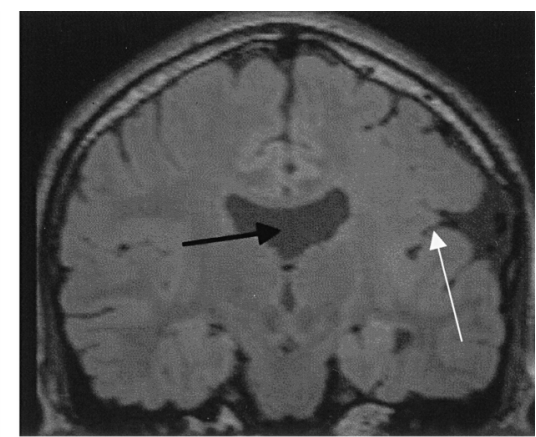

Fig. 11. A case of closed-lip schizencephaly. Abnormal and ectopic gray matter is seen in a $T_{1^{-}}$ weighted 3D-MP-RAGE image (arrow, a). Abnormal spots-such as high-signal areas (black arrows, b) - are scattered within the white matter surrounding the ectopic gray matter in $\mathrm{T}_{2}$-weighted 3D images (white arrow, b). With coronal-reformatted 3D-FLAIR contrast, the schizencephalic cleft is seen (white arrow, c), and the septum pellucidum is unclear (black arrow, c).

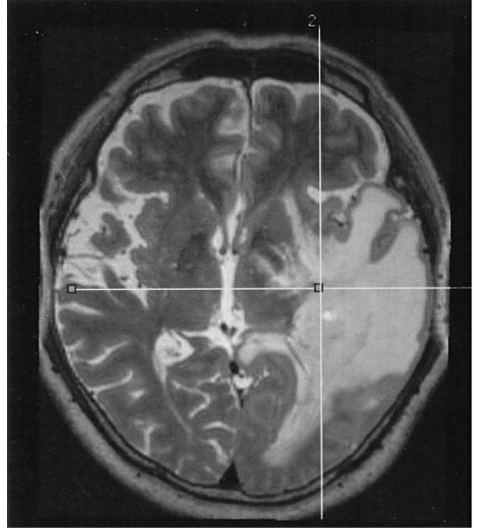

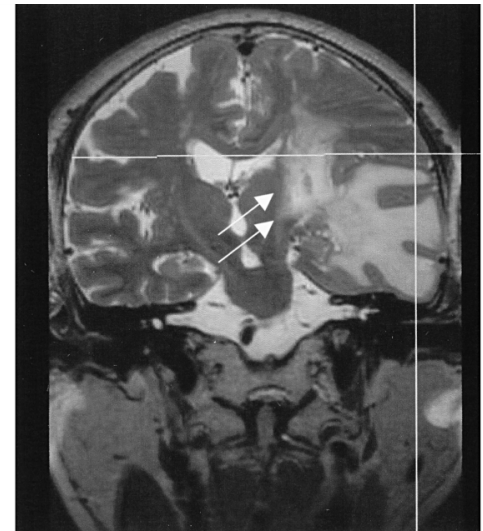

b

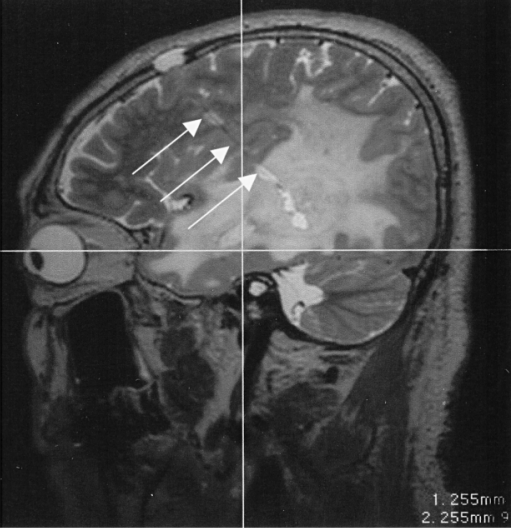

Fig. 12. A case of left temporal astrocytoma grade 3 (69-year-old man).

Axial (a), coronal (b), and sagittal (c) reformatted images. The corticospinal tract is easily visualized as a band of increased signal intensity (b, arrows). The biopsy tract is also visible (c, arrows).

struction from a single acquisition, but such long scans are susceptible to patient motion. With the current version of the software, 3D-TSE-VFL is not compatible with parallel imaging, although the high SNR at 3T would accommodate parallel imaging. With mSENSE or GRAPPA, scan time can be reduced by a factor of 2 to 3 , bringing 3DTSE-VFL scan times into a range similar to those of conventional 2D scans.

\section{Other new strategies}

One new technique is the spatially selective $3 \mathrm{D}$ slab with nonselective refocusing. ${ }^{10}$ The echo-train efficiency (i.e., the number of echoes collected per unit time during the echo train) of single-slab 3D spin-echo-based imaging can be increased significantly with the use of short, non-spatially selective RF pulses, instead of conventional sectionselective pulses, for both excitation and refocusing.
However, this approach sacrifices the ability to perform slab selection and thus substantially limits the range of clinical applications. The aims of the new method were to develop a simple and robust approach for spatially selective single-slab 3D turbo spin-echo (TSE) imaging that permits: (i) the use of nonselective refocusing RF pulses to achieve very short echo spacings and thus high echo-train efficiency; (ii) variable flip-angle refocusing RF pulses to be used without associated artifacts, and (iii) a high-quality slab profile to be achieved, thus minimizing the need to discard outer sections. An optimized excitation RF pulse yields a high-quality slab profile. Only the first echo spacing is extended to accommodate the duration of the excitation RF pulse; the remaining echo spacings are kept as short as possible under the constraints of gradient performance, resolution, and receiver bandwidth. This 
method allows the acquisition of a limited volume of the brain in a scan time shorter than that of a whole-brain scan.

Another new strategy is the use of longer echo trains. ${ }^{11}$ Tissue-specific prescribed signal evolutions, achieved by using refocusing RF pulses with variable flip angles, have been used to decrease the acquisition time for single-slab 3D turbo/fast spin-echo imaging by substantially increasing the usable echo-train duration compared to that achieved with $180^{\circ}$ refocusing RF pulses. With this technique, an echo-train duration of approximately one second yielded a brain image contrast similar to that achieved with conventional $\mathrm{T}_{2}$-weighted $2 \mathrm{D}$ spin-echo or 2D-TSE pulse sequences. Although previous studies have acquired at most one plane of three-dimensional $k$ space following each RF excitation pulse, it is possible, in principle, to acquire enough echoes during such long echo-train durations to encode two or more complete planes of $k$ space after each excitation, thus providing even faster 3D spin-echo-based acquisitions. This method would allow the use of more than 1,000 echoes per excitation, resulting in a four- to fivefold reduction in acquisition time.

In conclusion, 3D-TSE-VFL at 3T allowed us to perform high-resolution isotropic imaging of the whole brain in a clinically acceptable scan time with excellent contrast. With future reductions in scan time made possible with the higher signal-to-noise ratio, this technique will become part of the routine brain protocol at $3 \mathrm{~T}$.

\section{References}

1. Norris DG. High field human imaging. J Magn Reson Imaging 2003; 18:519-529.

2. Schwindt W, Kugel H, Bachmann R, et al. Magnetic resonance imaging protocols for examination of the neurocranium at 3 T. Eur Radiol 2003; 13: 2170-2179.

3. Schmitt F, Grosu D, Mohr C, et al. 3 Tesla MRI: successful results with higher field strengths. Radiologe 2004; 44:31-47.

4. Pruessmann KP, Weiger M, Scheidegger MB, Boesiger P. SENSE: sensitivity encoding for fast MRI. Magn Reson Med 1999; 42:952-962.

5. Schar M, Kozerke S, Fischer SE, Boesiger P. Cardiac SSFP imaging at 3 Tesla. Magn Reson Med 2004; 51:799-806.

6. Sodickson DK, Manning WJ, Lori NF, et al. Simultaneous acquisition of spatial harmonics (SMASH): fast imaging with radiofrequency coil arrays. Magn Reson Med 1997; 38:591-603.

7. Heidemann RM, Griswold MA, Haase A, Jakob PM. VD-AUTO-SMASH imaging. Magn Reson Med 2001; 45:1066-1074.
8. Griswold MA, Jakob PM, Heidemann RM, et al. Generalized autocalibrating partially parallel acquisitions (GRAPPA). Magn Reson Med 2002; 47:1202-1210.

9. Hennig J, Scheffler K. Hyperechoes. Magn Reson Med 2001; 46:6-12.

10. Mugler JP, Brookeman JR. Efficient spatiallyselective single-slab 3D turbo-spin-echo imaging. Proc Intl Soc Mag Reson Med 2004; 11:695.

11. Mugler JP, Brookeman JR. 3D turbo-spin-echo imaging with up to 1000 echoes per excitation: from faster acquisitions to echo-volumar imaging. Proc Intl Soc Mag Reson Med 2004; 11:2106.

12. Mugler JP, 3rd, Bao S, Mulkern RV, et al. Optimized single-slab three-dimensional spin-echo MR imaging of the brain. Radiology 2000; 216: 891-899.

13. Hennig J, Weigel M, Scheffler K. Multiecho sequences with variable refocusing flip angles: optimization of signal behavior using smooth transitions between pseudo steady states (TRAPS). Magn Reson Med 2003; 49:527-535.

14. De Coene B, Hajnal JV, Gatehouse P, et al. MR of the brain using fluid-attenuated inversion recovery (FLAIR) pulse sequences. AJNR 1992; 13:15551564.

15. Bakshi R, Kamran S, Kinkel PR, et al. Fluidattenuated inversion-recovery MR imaging in acute and subacute cerebral intraventricular hemorrhage. AJNR 1999; 20:629-636.

16. Maeda M, Yagishita A, Yamamoto T, Sakuma H, Takeda K. Abnormal hyperintensity within the subarachnoid space evaluated by fluid-attenuated inversion-recovery MR imaging: a spectrum of central nervous system diseases. Eur Radiol 2003; 13 (Suppl 4): L192-201.

17. Maeda M, Yamamoto T, Daimon S, et al. Arterial hyperintensity on fast fluid-attenuated inversion recovery images: a subtle finding for hyperacute stroke undetected by diffusion-weighted MR imaging. AJNR 2001; 22:632-636.

18. Maeda M, Koshimoto Y, Uematsu H, et al. Time course of arterial hyperintensity with fast fluidattenuated inversion-recovery imaging in acute and subacute middle cerebral arterial infarction. J Magn Reson Imaging 2001; 13:987-990.

19. Noguchi K, Ogawa T, Inugami A, et al. Acute subarachnoid hemorrhage: MR imaging with fluidattenuated inversion recovery pulse sequences. Radiology 1995; 196:773-777.

20. Noguchi K, Ogawa T, Seto H, et al. Subacute and chronic subarachnoid hemorrhage: diagnosis with fluid-attenuated inversion-recovery MR imaging. Radiology 1997; 203:257-262.

21. Hajnal JV, Oatridge A, Herlihy AH, Bydder GM. Reduction of CSF artifacts on FLAIR images by using adiabatic inversion pulses. AJNR 2001; 22: 317-322.

22. Herlihy AH, Hajnal JV, Curati WL, et al. Reduction of CSF and blood flow artifacts on FLAIR 
images of the brain with k-space reordered by inversion time at each slice position (KRISP). AJNR 2001; 22:896-904.

23. Herlihy AH, Oatridge A, Curati WL, Puri BK, Bydder GM, Hajnal JV. FLAIR imaging using nonselective inversion pulses combined with slice excitation order cycling and $\mathrm{k}$-space reordering to reduce flow artifacts. Magn Reson Med 2001; 46: 354-364.

24. Parizel PM, Dijkstra HA, Geenen GP, et al. Lowfield versus high-field MR imaging of the knee: a comparison of signal behaviour and diagnostic performance. Eur J Radiol 1995; 19:132-138.

25. Bakshi R, Caruthers SD, Janardhan V, Wasay M. Intraventricular CSF pulsation artifact on fast fluid-attenuated inversion-recovery MR images: analysis of 100 consecutive normal studies. AJNR 2000; 21:503-508.

26. Kallmes DF, Hui FK, Mugler JP, 3rd. Suppression of cerebrospinal fluid and blood flow artifacts in FLAIR MR imaging with a single-slab threedimensional pulse sequence: initial experience. Radiology 2001; 221:251-255.
27. Quencer RM, Pattany PM. Fluid-attenuated inversion recovery now with another acronym: "KRISP FLAIR". AJNR 2001; 22:805-806.

28. Tanaka N, Abe T, Kojima K, Nishimura $H$, Hayabuchi N. Applicability and advantages of flow artifact-insensitive fluid-attenuated inversionrecovery MR sequences for imaging the posterior fossa. AJNR 2000; 21:1095-1098.

29. Wu HM, Yousem DM, Chung HW, Guo WY, Chang CY, Chen CY. Influence of imaging parameters on high-intensity cerebrospinal fluid artifacts in fast-FLAIR MR imaging. AJNR 2002; 23:393-399.

30. Naganawa S, Koshikawa T, Nakamura T, et al. Comparison of flow artifacts between 2D-FLAIR and 3D-FLAIR sequences at 3 T. Eur Radiol 2004; 14:1901-1908.

31. Tan IL, van Schijndel RA, Pouwels PJ, Ader HJ, Barkhof F. Serial isotropic three-dimensional fast FLAIR imaging: using image registration and subtraction to reveal active multiple sclerosis lesions. AJR 2002; 179:777-782. 